\title{
Whole-body vibration exercise in postmenopausal osteoporosis
}

\author{
Magdalena Weber-Rajek ${ }^{1}$, Jan Mieszkowski ${ }^{2}$, Bartłomiej Niespodziński ${ }^{2}, K^{2}$ atarzyna Ciechanowska ${ }^{1}$ \\ ${ }^{1}$ Department of Laser Therapy and Physiotherapy, Ludwik Rydygier Collegium Medicum in Bydgoszcz, Nicolaus Copernicus University \\ in Torun, Poland \\ ${ }^{2}$ Institute of Physical Culture, Kazimierz Wielki University in Bydgoszcz, Poland
}

\begin{abstract}
The report of the World Health Organization (WHO) of 2008 defines osteoporosis as a disease characterized by low bone mass and an increased risk of fracture. Postmenopausal osteoporosis is connected to the decrease in estrogens concentration as a result of malfunction of endocrine ovarian function. Low estrogens concentration causes increase in bone demineralization and results in osteoporosis. Physical activity, as a component of therapy of patients with osteoporosis, has been used for a long time now. One of the forms of safe physical activity is the vibration training. Training is to maintain a static position or execution of specific exercises involving the appropriate muscles on a vibrating platform, the mechanical vibrations are transmitted to the body of the patient. According to the piezoelectric theory, pressure induces bone formation in the electrical potential difference, which acts as a stimulant of the process of bone formation. Whole body vibration increases the level of growth hormone and testosterone in serum, preventing sarcopenia and osteoporosis. The aim of this study was to review the literature on vibration exercise in patients with postmenopausal osteoporosis based on the PubMed and Medline database. While searching the database, the following key words were used 'postmenopausal osteoporosis' and 'whole-body vibration exercise'.
\end{abstract}

Key words: postmenopausal osteoporosis, whole-body vibration exercise.

\section{Introduction}

Osteoporosis, a systemic skeletal system disease affecting patients in different ages characterized by a significant increase in fractures likelihood due to a decrease in bone mineral density (BMD) and abnormal bone microarchitecture [1]. Osteoporosis is mostly observed in female population, where a significant increase in incidence is recorded after menopause. This specific type of osteoporosis is being called postmenopausal, and is mainly caused by the cessation of the endocrine ovarian function. Estrogen deficiency exacerbates bone demineralization processes resulting in bone abnormal microarchitecture. Postmenopausal osteoporosis treatment in the $21^{\text {st }}$ century has evolved focusing on prevention, screening, diagnosis and early and very specified therapy. However, still treatment of this disease remains a long-term process, not giving $100 \%$ guarantee for inhibition of its development. Many medicines that can be administered orally or parenterally, have a different mechanism of action, and can cause more problems when deciding to initiate treatment. This confirms the need for specific recommendations on how, when and who to treat with post-menopausal osteoporosis in accordance with the principles of evidence-based medicine [2]. An indispensable element in the treatment of osteoporosis should be physiotherapy, which if used professionally and systematically improves the quality of life of patients and reduces the risk of main causes of fractures, which result from falling. Among the physical methods, various forms of physical activity deserve special attention $[3,4]$. In patients with osteoporosis due to an increased risk of fall, the physical activity must be first of all safe. One of the forms of safe physical activity is the vibration training. Training is to maintain a static position or execution of specific exercises involving the appropriate muscles on a vibrating platform, the mechanical vibrations are transmitted to the body of the patient [5].

According to the piezoelectric theory, pressure induces bone formation in the electrical potential difference, which acts as a stimulant of the process of bone formation. A stronger stimulator of osteogenesis is the effort performed in the upright position than in the horizontal, which explains the good effects of the vibration therapy. Whole-body vibration (WBV) increases the level of growth hormone and testosterone in serum, preventing sarcopenia and osteoporosis [6]. Vibration exercise also increases muscle strength and neuromuscular coordination, which in the case of patients with

Corresponding author: Magdalena Weber-Rajek, Department of Laser Therapy and Physiotherapy, Ludwik Rydygier Collegium Medicum in Bydgoszcz, Nicolaus Copernicus University in Torun, 9 M. Curie Skłodowskiej St., 85-094 Bydgoszcz, Poland, e-mail: magdawr69@gmail.com

To cite this article: Weber-Rajek M, Mieszkowski J, Niespodziński B, Ciechanowska K. Whole-body vibration exercise in postmenopausal osteoporosis. Prz Menopauzalny 2015; 14: 41-47.

Submitted: 8.05.2014; Accepted: 18.11.2014 
osteoporosis can reduce the risk of falls resulting in broken bones. Whole-body vibration is also one of the elements of the ICARO (Innovative Comprehensive Active Rehabilitation of Osteoporosis) strategy [7].

Despite its effectiveness still many studies not fully determined the correct vibration frequency range which is most effective. In animal studies, there is also no consensus as to the frequency of vibration. Flieger et al. [8] gave the vibration treatment to postmenopausal female rabbits. Experimental groups were exposed to vibration at a frequency of $50 \mathrm{~Hz}$ and acceleration $20 \mathrm{~m} / \mathrm{s}^{2}$, for 5 weeks, about half an hour a day. In relation to the control group in the exhibited animals a significant increase in BMD $(p<0.05)$ was observed. Oxlund et al. [9] compared the effect of different frequencies of vibration on the process of bone formation in animals and demonstrated that the frequency of $45 \mathrm{~Hz}$ most effectively stimulates osteoblasts division and inhibits bone resorption (vibrations were applied daily for 90 days for 30 minutes). Wolf et al. [10] in experimental studies, used low-frequency vibrations to stimulate healing of bones in sheep. Six sheep were exposed to vibration at a frequency of $20 \mathrm{~Hz}$ and an amplitude of $2 \mathrm{~mm}$ for eight weeks. Between the exposed and the control group there were no significant differences, however, in $11 \%$ of the exposed group, adhesions were stronger. Chen et al. [11] conducted an experiment on 76 rabbits after fracture of the radius. Rabbits were divided into six groups. Five groups were exposed to vibration with frequencies of $12.5 \mathrm{~Hz} ; 25 \mathrm{~Hz} ; 50 \mathrm{~Hz}$; $100 \mathrm{~Hz}$ and $200 \mathrm{~Hz}$. The analysis of the results showed that the rate of healing for exposed groups does not depend on the frequency, but it is statistically higher than in the control group. The strength tests showed that animals exposed to the fracturing force are from $20 \%$ to $30 \%$ higher in comparison to the control group. Analyses showed that the value of a fracturing force depends on the frequency and adopts the largest value for $25 \mathrm{~Hz}$, than for $50 \mathrm{~Hz}, 12.5 \mathrm{~Hz}, 100 \mathrm{~Hz}$ and $200 \mathrm{~Hz}$.

The aim of this study was to review the literature on vibration exercise in patients with postmenopausal osteoporosis based on the PubMed and Medline database. While searching the database, the following key words were used 'postmenopausal osteoporosis' and 'whole-body vibration exercise'.

\section{Review of research}

The first researches on the impact of vibration on the process of bone formation were carried out on animals [8-12]. Over the last ten years, the interest of this form of the therapy for osteoporotic changes has increased; that is why a large number of publications on the human model were performed. Many of studies evaluate the effectiveness of this type of therapy especially in the increase in bone density, some of them concern the analgesic effect and other put the importance of its influence on balance and overall fitness.

\section{Whole-body vibration exercise and bone density}

Studies concerning the bone density effect mostly demonstrate an increase in BMD, depending on the duration and vibration frequencies. In order to investigate the effect of vibration on post-menopausal bone loss Rubin et al. [13] used a vibration frequency of $30 \mathrm{~Hz}$ and acceleration of $2 \mathrm{~m} / \mathrm{s}$. Each of the 70 women tested twice daily $(2 \times 10$ minutes) by 12 months was subjected to WBV. A decrease in bone loss by $1.5 \%$ was diagnosed in the spine and $2.17 \%$ at the femoral neck region. It was noted that the greatest benefit from the use of vibration was provided to women weighing less than $65 \mathrm{~kg}$, who obtained an increase in BMD by 3.35\%. Ruan et al. [14] assessed the impact of the therapy on the vibration platform in postmenopausal women with osteoporosis. In the study, 116 women were divided into two groups. In the first group, the vibration platform therapy was applied (frequency of vibration $30 \mathrm{~Hz}$, amplitude of $5 \mathrm{~mm}$ ). Treatment lasted for 10 minutes, 5 times a week for 6 months. The patients of the second group and the control group were not subjected to any treatment. Before the beginning of the study and after the completion, BMD was studied in both groups. In the treated group after 3 months, BMD of the lumbar region increased by $1.3 \%$ and $4.3 \%$ in the sixth month. Bone mineral density in the control group decreased in the third month, but the results were not statistically significant. In the sixth month BMD decreased in this group by $1.9 \%$. In the research group, BMD of the femoral neck region increased slightly after the vibration therapy after 3 months, but without statistical significance. In the sixth month BMD increased by $3.2 \%$. In the control group BMD was not significantly reduced in the third month, but decreased significantly in the sixth month $(1.7 \%)$ compared with the baseline results. In the treated vibratory group also chronic back pain decreased, which was assessed by the Visual Analogue Scale (VAS).

A similar experiment was carried out by Rubin et al. [15] in order to investigate the effect of vibration on post-menopausal bone loss. Researchers used a vibration frequency of $30 \mathrm{~Hz}$ and acceleration of $2 \mathrm{~m} / \mathrm{s}$. Each of the 70 women tested was subjected to WBV twice daily ( $2 \times 10$ minutes) for 12 months. A decrease in bone loss by $1.5 \%$ was diagnosed in the spine and $2.17 \%$ at the femoral neck region. It was noted that the greatest benefit from the use of vibration was enjoyed by women weighing less than $65 \mathrm{~kg}$, who obtained an increase in BMD by $3.35 \%$.

Furthermore, Verschueren et al. [16] carried out 6 months' vibratory exercise experiment on a group 
of 21 postmenopausal women (58-74 years old) who performed five different exercises involving the muscles of the lower extremities. Gradually the load of vibration exercise was increased by the oscillation frequency (35-40 Hz), the amplitude of motion (1.7-2.5 mm), the number and exercise time (max. 30 minutes). The study showed an increase in bone density of the hip bone of $0.93 \%$ after 6 months of vibration exercise. Similar conclusions were presented in a work of Lai et al. [17]. The experiment was performed on a group of 28 women with vibroacoustic therapy $(30 \mathrm{~Hz}, 3$ times per week, 5 minutes, 6 months of therapy). The studies showed an increase in BMD of $2.032 \%(p=0.047)$, whereas in the control group it decreased by $0.046 \%(p=0.188)$. Similar conclusions were shown in the work of Von Stengel et al. [18]. They executed the experiment on 108 postmenopausal women, randomly assigned to 3 groups. The first group underwent vibrating therapy (12.5 Hz, amplitude of $12 \mathrm{~mm}$, three 15 minutes' sessions per week) connected with the dynamic exercises of deep knee bends, second group - only vibrational therapy $(35 \mathrm{~Hz}$, amplitude of $1.7 \mathrm{~mm}$, three sessions per week, for 15 minutes), third group - performed low-intensity exercises. In all groups at baseline and at 12 months of training, BMD in the hip and spine areas were measured. The results showed an increase in BMD at the lumbar spine area in vibrational therapy groups.

Results of meta-analysis of the effects of the vibration therapy on BMD in postmenopausal women, young adults and children done by Slatkowska et al. [19] showed similar conclusions to those presented above, that is, postmenopausal women - an increase in hip BMD; no increase in BMD and the tibia, young adults - no increase in BMD of the spine and hip, children and young people - a significant increase in BMD of the spine and tibia.

\section{Whole-body vibration exercise influence on the balance state and overall fitness}

A large group of studies indicate an influence of vibration therapy not only on BMD but focus on balance control and overall fitness (health-promoting influence). Gusi et al. [20] reported that studies on a group of 28 postmenopausal women, randomly divided into two groups - first group treated with the vibrational therapy $(12.6 \mathrm{~Hz})$, second group walking $(55$ minutes of walking and 5 minutes of stretching). Both experimental programs consisted of three sessions per week for 8 months. In both groups, BMD in the area of the lumbar spine and hip was examined and a balance test was performed. After 8 months, BMD at the femoral neck area in a vibratory treated group increased by $4.3 \%$ $(p=0.011)$ compared to the group who performed walking. In contrast, BMD at the lumbar spine region remained unchanged in both groups. The balance im- proved in vibration treated group by $29 \%$. The group which performed walking showed no improvement in balance. This clearly indicates the beneficial influence of this type of therapy on general health.

Similar conclusions were shown in a work of Stolzenberg et al. [21]. They compared the efficacy of classical balance training (BAL) and WBV on postural control in post-menopausal women with low bone density. In the study of 68 patients of which 57 completed the nine-month therapy. All patients had resistive exercise. They were randomized into two groups, the BAL $(n=31)$ performing progressive balance and coordination exercise and WBV-group $(n=26)$ performing vibration exercise for four minutes (from 24 to $26 \mathrm{~Hz}$ and from 4 to $8 \mathrm{~mm}$ range) on the Galileo Fitness. Each month, the performance of a single leg stance exercise on a standard unstable surface (Posturomed) was tested. Single leg stance, Romberg-stance, semi-tandemstance and tandem-stance were tested on a ground reaction force platform (Leonardo) twice, at the beginning and the end of the study. The speed of movement on the Posturomed improved by $28.3(36.1 \%)(p<0.001)$ in the WBV-group and $18.5(31.5 \%)(p<0.001)$ in the BAL-group by the end of the nine-month period, but there were no differences noted between the two groups $(p=0.45)$. Further studies of the same authors [22] noted that WBV lasting for a short time can improve the neuromuscular function in post-menopausal women with low bone density than proprioceptive training (countermovement jump, multiple hopping and sit-to-stand tests).

Furthermore, Klarner et al. [23] studied postmenopausal women and the effects of various kinds of WBV exercises on neuromuscular performance and body composition. In the Erlangen Longitudinal Vibration Study II (ELVIS-II-Study), 108 postmenopausal women between 60 and 75 years of age were selected and divided into three groups. Two WBV training-groups ( $n=36$ each) performed an identical exercise three times a week for 15 minutes on two different types of vibration surfaces for a period of one year: vertical vibrating, $35 \mathrm{~Hz} ; 1.7 \mathrm{~mm}$ (VG) and rotational vibrating $12.5 \mathrm{~Hz}$; $12 \mathrm{~mm}(\mathrm{RG})$. A control group $(n=36)$ performed a low intensity exercise involving relaxation and gymnastics. Muscular strength and power increased. Body composition was determined by dual-energy X-ray absorptiometry (DXA). Patients answered the questions in a questionnaire concerning pain intensity. According to the study, maximum leg strength (VG: 24.4\%; RG: 26.6\%; KG: $6.2 \% ; p<0.001)$ and maximum trunk flexion strength (VG: $12.2 \%$; RG: $11.5 \%$; KG: $-5.5 \% ; p=0.01$ ) significantly increased in WBV groups (control group no significant changes). It was noted that there were no changes in body composition parameters such as lean body mass, appendicular muscle mass, fat mass in all analyzed groups, but both WBV groups reported a sig- 
nificant decrease in pain intensity in the large joints $(p<0.05)$. This study clearly indicates similar results about pain intensity after WBV therapy in postmenopausal women presented by Ruan et al. [14]. This beneficial effect is very important in improving the quality of life of osteoporotic patients.

Time of the WBV therapy is one of the most important elements of its success, short time of the therapy can be connected to its limited effectiveness. GómezCabello et al. [24] studied 49 old people, 20 men and 29 women. They were randomized into WBV group or control group. A group of 24 elderly exercised on a vibration platform 3 times a week for 11 weeks. With the use of dual-energy X-ray absorptiometry and peripheral quantitative computed tomography bone-related variables were assessed. The effects of the intervention on the bone-related variables and the changes within the group throughout the intervention period were determined with the use of two-way repeated measures one-way analysis of variance (group by time). The differences between groups for bone-related variables in pre- and post-training assessments and in the percentage of change between groups were tested by analysis of covariance. Age, height, subtotal lean mass and daily calcium intake as covariates were analyzed. Bone mineral content and density measured by dual-energy $\mathrm{X}$-ray absorptiometry did not show any changes. Wholebody vibration training revealed no changes. Bone mineral density decreased significantly in the whole-body vibration group (all $p<0.05$ ). The study concluded that a short-duration WBV therapy is not enough to change bone mineral content or BMD. In elderly people it causes minimal changes in bone structure.

Whole-body vibration exercise can be a useful addition to pharmacological therapy during osteoporosis. Iwamoto et al. [25] studied a group of postmenopausal women (aged 51-91) with osteoporosis over a period of 6 months treated with WBV. The study group of women was receiving alendronate and furthermore was divided into two groups: an exercise group (WBV twice weekly for 4 minutes) and a control group (no exercises). After 6 months of the WBV exercise, the results showed that flexibility, body balance, and walking velocity were significantly improved in the exercise group compared with the control group. It was noted that the reductions in serum alkaline phosphatase and urinary cross-linked $N$-terminal telopeptides of type I collagen during the 6-month period were comparable between the two groups. The study highlighted the beneficial effects of WBV exercises to improve physical function in postmenopausal osteoporotic women treated with alendronate. And it clearly indicated that this type of therapy can be a useful tool in restoring efficiency. Similar studies were conducted by Iwamoto et al. [26]. They studied the influence of WBV exercises on the reduction of alendronate on BMD and chronic back pain in women with osteoporosis over a period of 12 months. The main aim of this study was to ascertain whether WBV would improve the effect of alendronate on lumbar BMD and bone replacement, and reduce chronic back pain. In the study, 50 post-menopausal women with osteoporosis (aged 55-88) which were divided into 2 groups of 25 patients each. The first group took $5 \mathrm{mg}$ alendronate daily (ALN) and the second one took alendronate plus exercise (ALN + EX). The WBV on a Galileo machine had an intensity of $20 \mathrm{~Hz}$ weekly for 4 minutes. Lumbar BMD was measured by dual energy X-ray absorptiometry (Hologic QDR 1500 W). Urinary cross-linked $\mathrm{N}$-terminal telopeptides of type I collagen (NTX) and serum alkaline phosphatase (ALP) levels were measured by enzyme-linked immunosorbent assay and standard laboratory techniques, respectively. Chronic back pain was evaluated by face scale score at baseline and every 6 months. According to the study, it was noted that there were no significant differences in baseline characteristics, including age, body mass index, years since menopause, lumbar BMD, urinary NTX and serum ALP levels, or face scale score between the two groups. The increase in lumbar BMD and the reduction in urinary NTX and serum ALP levels were similar in the ALN and ALN + EX groups. In contrast, the reduction in chronic back pain was larger in the ALN + EX group than in the ALN group. The study appears to be a major breakthrough in the treatment of reducing chronic back pain by the use of a Galileo machine. The probability is the effect of relaxing the back muscles by in post-menopausal osteoporotic women treated with alendronate.

\section{Whole-body vibration exercise - bone mineral density conflicting results}

According to Bemben et al. [27], WBV effect on BMD is not as clear in humans as in animal models. They examined 55 postmenopausal women with estrogen-deficiency who performed the WBV and resistance training on BMD and bone metabolism over an 8 months' period. The patients were divided into three groups: a resistance training group $-\mathrm{R}(n=22)$, a WBV plus resistance training group - WBVR $(n=21)$, and a control group - CON $(n=12)$. In the study, R and WBVR groups performed upper and lower body resistance exercises 3 days/week at $80 \% 1$ repetition maximum (1RM). WBVR received vibration $(30-40 \mathrm{~Hz}, 2-2.8 \mathrm{~g}$ ) in three different positions preceding the resistance exercises. At baseline and after the study, daily calcium intake, bone markers (ALP); C-terminal telopeptide of type I collagen (CTX), and BMD of the spine, dual femur, forearm, and total body (DXA) were measured. It was noted that at baseline, there were no significant group differences in strength, BMD, or bone marker variables. After 8 months of R or WBVR, there were no significant group or time effects in ALP, CTX, or total body, spine, 
left hip or right trochanter BMD. However, right total hip and right femoral neck BMD significantly $(p<0.05)$ decreased in all groups. A group $x$ time interaction $(p<0.05)$ was detected at radius 33\% BMD site, with CON slightly increasing, and WBVR slightly decreasing. $R$ and WBVR significantly $(p<0.05)$ increased $1 R M$ strength for all exercises, while CON generally maintained strength. WBVR had significantly $(p<0.05)$ greater percent increases in muscular strength than $R$ at 4 months for lat pull-down, seated row, hip abduction and hip adduction and at 8 months for lat pull-down, hip abduction and hip adduction. Bone metabolism was not affected by resistance training in both groups with or without WBV and extra WBV increased the positive effects of resistance training on muscular strength in women.

Beck et al. [28] also noted that humans were not examined as the animal models and low versus high intensity WBV was unknown. The WBV was an alternative therapy for loss of musculoskeletal competence in old people. The main aim of the 8 months' study was to assess the effect of low and higher intensity WBV on risk factors for hip fracture in 47 postmenopausal women. The study was randomized and examined the influence of low-intensity WBV $(15$ minutes, $30 \mathrm{~Hz}$, $0.3 \mathrm{~g} /$ twice a week) or higher intensity WBV $(2 \times 3 \mathrm{minu}$ tes, $12.5 \mathrm{~Hz}, 1 \mathrm{~g}$ ) on anthropometrics, bone (whole body, hip, spine, forearm, and heel), muscle (wall squat and chair rise), and balance (tandem walk and single leg stance). Physical activity, daily calcium, and compliance were measured. Effects were examined by repeatedmeasures analysis of covariance, controlling for age, height, weight, calcium, physical activity, compliance, and baseline values. There were no differences in both groups in any measure at 8 months, but within-group effects were evident. Controls lost bone at the trochanter $(-6 \%, p=0.03)$ and lumbar spine $(-6.6 \%, p=0.02)$, whereas WBV groups did not. Whole-body vibration patients improved wall squat (up to $120 \%, p=0.004$ ) and chair rise performance (up to $10.5 \%, p=0.05$ ). Over a period of 8 months of twice-weekly WBV may reduce bone loss at the hip and spine and improve lower limb muscle function. These changes may reduce the risk of falls and hip fracture. Slatkowska et al. [29] conducted a study in a group of 202 healthy postmenopausal women. Patients were randomly allocated into three groups: two research groups and one control. Patients from the research groups were subjected to vibrational therapy $(0.3 \mathrm{~g}, 90 \mathrm{~Hz}$ or $30 \mathrm{~Hz}, 20$ minutes per day, for a period of 12 months). All study participants received additionally vitamin D and calcium. The studies found no effects of the vibration therapy on BMD change in postmenopausal women who received calcium and vitamin D.

\section{Discussion}

Most of the in vitro research have demonstrated positive effects of WBV therapy on bone remodeling in case of postmenopausal patients. These conclusions are based on the theory that WBV exercise therapy as a form of physical activity can regulate bone maintenance and stimulate bone formation including the accumulation of mineral, in addition to strengthening muscles, improving balance, and thus reducing the overall risk of falls and fractures (as a primary result of osteoporosis and its consequences). Sadly this theory may be undermined due to the lack of changes in the activity of many bone turnerover markers as a result only of physical activity. It may bring significant doubts about influence on the processes of resorption and bone formation. In addition it is very important to remember that positive results can be indicated only in situation of a bone remodeling misbalance as a result of postmenopausal osteoporosis or any other osteological diseases. Similar conclusions were shown in work of Slatkowska et al. [29]. They found no changes in BMD in postmenopausal women who were subjected to WBV. What draws the attention is the fact that post-menopausal patients qualified for this study were healthy (had no osteoporotic changes), which might suggest that this form of therapy is more effective for bone tissue of a reduced density. At the same time we read that in Gomez-Cabello's work [24], a decrease of bone mineral density was observed. Bone mineral density decrease in the group in which vibrations were performed as well as in the groups practicing without vibrations and the control one were observed also in Bemben's work [27]. In Iwamoto's research [25, 26] the BMD increase in the group using vibration exercises was identical as in the non-exercising group and simply stemmed out from simultaneous aldosterone treatment in both groups. Thus, the research results are not at all explicit.

Others researches of Slatkowska et al. [19] gave us the conclusions that vibration training may cause a significant increase in bone density in children, increase in bone density in postmenopausal women, and increase bone density in young adults. In addition, it is difficult to answer the question whether the effectiveness of WBV is comparable or higher than other forms of physical activity. According to Borer et al. [30], Srinivasan et al. [31], Czarkowska-Pączek et al. [32], to receive a positive effect of the exercise in postmenopausal women it should be dynamic, exceed a threshold intensity, exceed a threshold strain frequency, be relatively brief and the duration period of bone formation and remodeling should be conducted in the treatment of long time. The minimal period after which BMD can be evaluated and the results of physical activity (including WBV) on bone remodeling can be established is 12 
months. Therefore the conclusions shown in GómezCabello et al. [24] studies are inappropriate. They analyzed influence of vibration exercises on BMD carried out only for an 11 weeks' period, therefore the increase of BMD was not achieved.

Whole-body vibration therapy similar to the other forms of activity effects depend on its intensity, and used frequency. Vibration frequency which was used in presented studies were $12.5 \mathrm{~Hz}, 20 \mathrm{~Hz}, 30 \mathrm{~Hz}$, $40 \mathrm{~Hz}$ and $90 \mathrm{~Hz}$. Klaner et al. studies [23] compared the efficacy of treatment of the vibration at different frequencies $(12.5 \mathrm{~Hz}$ and $30 \mathrm{~Hz}$ ), and demonstrated the efficacy of both frequencies without significant differences between groups. In view of inconsistent results, it is difficult to determine what range of used frequencies would be the most appropriate to obtain positive effects of the therapy. However, it is always necessary to be remember that each time therapy should be started from the lower vibration frequencies gradually increasing them along with the duration of therapy time [33].

Vibration training can bring success in treating pain resulting from osteoporosis and it increases physical fitness. Increasing muscle strength, neuromuscular coordination and balance, reducing the risk of falls, often resulting in fractures. Noteworthy research of Iwamoto et al. $[25,26]$, in which women with postmenopausal osteoporosis were treated with alendronate and combination therapy (alendronate and WBV). In both groups, a similar increase in BMD was found, however, in women treated with the combination therapy, chronic back pain was reduced. The analgesic effect of WBV is also confirmed by Klarner et al. [23] and Ruan Xiang-yan et al. [14]. Beneficial effects on the improvement of physical fitness after the application of vibrational therapy is confirmed by studies of Beck et al. [28], Bemben et al. [27], Von Stengel et al. [18] and Gusi et al. [20]. Noteworthy, Rubin et al. [13] research showed that the greatest benefit from the use of vibration were obtained by women weighing less than $65 \mathrm{~kg}$. The process of bone formation is a long process because in most of the studies cited, the therapy lasted 6-12 months.

\section{Conclusions}

Accurate clarification is needed to determine the relationships between biochemical factors, bone structure and effectiveness parameters of the vibration therapy for osteoporosis and associated threats.

Whole-body vibration exercise therapy is only one of the types of physical activity recommended for the treatment of postmenopausal osteoporosis and cannot be used as substitute therapy for pharmacological and dietary treatment methods. But it may complement already known and described postmenopausal treatment methods and bring tangible results in therapy.

\section{Disclosure}

Authors report no conflict of interests.

\section{References}

1. Kanis JA, on behalf of the World Health Organization Scientific Group (2008). Assessment of osteoporosis at the primary health-care level. Technical Report. WHO Collaborating Centre, University of Sheffield, UK.

2. Pawlak-Buś K, Leszczyński P. Farmakoterapia parenteralna i doustna w zapobieganiu złamaniom w przebiegu osteoporozy pomenopauzalnej. Prz Menopauzalny 2013; 12: 271-277.

3. Delecluse C, Roelants M, Verschueren S. Strength increase after wholebody vibration compared with resistance training. Med Sci Sports Exerc 2003; 35: 1033-1041.

4. Grześkowiak M, Leszczyński P, Lewandowski J. Postępowanie fizjoterapeutyczne u pacjentów z osteoporozą bez złamań i ze złamaniami. Prz Menopauzalny 2013; 12: 142-145.

5. Runge $M$, Rehfeld $G$, Resnicek $E$. Balance training and exercise in geriatric patients. J Musculoskelet Neuron Interact 2000; 1: 61-65.

6. Cardinale M, Pope MH. The effects of whole body vibration on humans: dangerous or advantageous? Acta Physiol Hung 2003; 90: 195-206.

7. Deszczyński J, Bronikowski A, Nagraba Ł, et al. Innowacyjna, Kompleksowa, Aktywna, Rehabilitacja Osteoporozy nową strategią leczenia i profilaktyki. Artroskopia i Chirurgia Stawów 2010; 6: 29-35.

8. Flieger J, Karachalios T, Khaldi L, et al. Mechanical stimulation in the form of vibration prevents postmenopausal bone loss in ovariectomized rats. Calcif Tissue Int 1998; 63: 510-514.

9. Oxlund BS, Ortoft G, Andreassen TT. Low-intensity, high-frequency vibration appears to prevent the decrease in strength of the femur and tibia associated with ovariectomy of adult rats. Bone 2003; 32: 69-77.

10. Wolf S, Augat P, Eckert-Hübner K, et al. Effects of high-frequency, lowmagnitude mechanical stimulus on bone healing. Clin Orthop Relat Res 2001; 385: 192-198.

11. Chen LP, Han ZB, Yang XZ. The effects of frequency of mechanical vibration on experimental fracture healing. Zhonghua Wai Ke Za Zhi 1994; 32: 217-219.

12. Chen LP, Han ZB, Yang XZ. Experimental study of fracture healing promotion with mechanical vibration in rabbits. Zhonghua Wai Ke Za Zhi 1994; 32: 215-216.

13. Rubin C, Turner AS, Bain S. Anabolism. Low mechanical signals strengthen long bones. Nature 2001; 412: 603-604.

14. Ruan XY, Jin FY, Liu YL, et al. Effects of vibration therapy on bone mineral density in postmenopausal women with osteoporosis. Chin Med J (Engl) 2008; 121: 1155-1158.

15. Rubin C, Recker R, Cullen D. Prevention of postmenopausal bone loss by a low-magnitude, high-frequency mechanical stymuli: a clinical trial assessing compliance, efficacy and safety. J Bone Miner Res 2004; 19: 343-351.

16. Verschueren SM, Roelants M, Delecluse C, et al. Effect of 6-month whole body vibration training on hip density, muscle strength, and postural control in postmenopausal women: a randomized pilot study. J Bone Miner Res 2004; 3: 352-359.

17. Lai CL, Tseng SY, Chen CN, et al. Effect of 6 months of whole body vibration on lumbar spine bone density in postmenopausal women: a randomized controlled trial. Clin Interv Aging 2013; 8: 1603-1609.

18. Von Stengel S, Kemmler W, Bebenek M, et al. Effects of whole-body vibration training on different devices on bone mineral density. Med Sci Sports Exerc 2011; 43: 1071-1079.

19. Slatkovska L, Alibhai SM, Beyene J, et al. Effect of whole-body vibration on BMD: a systematic review and meta-analysis. Osteoporos Int 2010; 21: 1969-1980.

20. Gusi N, Raimundo A, Leal A. Low-frequency vibratory exercise reduces the risk of bone fracture more than walking: a randomized controlled trial. BMC Musculoskelet Disord 2006; 7: 92.

21. Stolzenberg N, Belavý DL, Rawer R, et al. Whole-body vibration versus proprioceptive training on postural control in post-menopausal osteopenic women. Gait Posture 2013; 38: 416-420.

22. Stolzenberg N, Belavý DL, Rawer R, et al. Vibration or balance training on neuromuscular performance in osteopenic women. Int J Sports Med 2013; 34: 956-962. 
23. Klarner A, von Stengel S, Kemmler W, et al. Effects of two different types of whole body vibration on neuromuscular performance and body composition in postmenopausal women. Dtsch Med Wochenschr 2011; 136: 2133-2139.

24. Gómez-Cabello A, González-Agüero A, Morales S, et al. Effects of a short term whole body vibration intervention on bone mass and structure in elderly people. J Sci Med Sport 2014; 17: 160-164.

25. Iwamoto J, Sato Y, Takeda T, et al. Whole body vibration exercise improves body balance and walking velocity in postmenopausal osteoporotic women treated with alendronate: Galileo and Alendronate Intervention Trail (GAIT). Musculoskelet Neuronal Interact 2012; 12 136-143.

26. Iwamoto J, Takeda T, Sato Y, et al. Effect of whole-body vibration exer cise on lumbar bone mineral density, bone turnover, and chronic back pain in post-menopausal osteoporotic women treated with alendronate. Aging Clin Exp Res 2005; 17: 157-163.

27. Bemben DA, Palmer IJ, Bemben MG, et al. Effects of combined wholebody vibration and resistance training on muscular strength and bone metabolism in postmenopausal women. Bone 2010; 47: 650-656.
28. Beck BR, Norling TL. The effect of 8 mos of twice-weekly low- or higher intensity whole body vibration on risk factors for postmenopausal hip fracture. Am J Phys Med Rehabil 2010; 89: 997-1009.

29. Slatkovska L, Alibhai SM, Beyene J, et al. Effect of 12 months of wholebody vibration therapy on bone density and structure in postmenopausal women: a randomized trial. Ann Intern Med 2011; 155: 668-679.

30. Borer KT, Fogleman K, Gross K, et al. Walking intensity for postmenopausal bone mineral preservation and accrual. Bone 2007; 41: 713-721.

31. Srinivasan S, Weimer DA, Agans SC, et al. Low-magnitude mechanical loading becomes osteogenic when rest is inserted between each load cycle. J Bone Miner Res 2002; 17: 1613-1620.

32. Czarkowska-Pączek B, Wesołowska K, Przybylski J. Wysiłek fizyczny w profilaktyce osteoporozy. Przegl Lek 2011; 68: 103-106.

33. Borer KT. Physical activity in the prevention and amelioration of osteoporosis in women: interaction of mechanical, hormonal and dietary factors. Sports Med 2005; 35: 779-830. 